\title{
Endogenous antigen presentation of MHC class II epitopes through non-autophagic pathways
}

\author{
Carol S. K. Leung * \\ Department of Haematology, University College London Cancer Institute, University College London, London, UK
}

Antigenic peptides presented by major histocompatibility complex (MHC) class II molecules are generally derived from exogenous proteins acquired by antigen presenting cells. However, in some circumstances, MHC class II molecules can present intracellular proteins expressed within the antigen-presenting cells. There are several described pathways by which endogenous antigens are degraded and gain access to $\mathrm{MHC}$ class II molecules. These include autophagy and other non-autophagic pathways; the latter category includes the MHC class I-like pathways, heat shock protein 90-mediated pathways, and internalization from the plasma membrane. This review will summarize and discuss the non-autophagic pathways.

Edited by:

Monique Gannagé,

University of Geneva, Switzerland

Keywords: antigen presentation, MHC class II molecules, endogenous presentation, intracellular antigens, non-classical pathways

Reviewed by:

Justine Mintern,

University of Melbourne, Australia

Laurence C. Eisenlohr,

Thomas Jefferson University, USA

*Correspondence:

Carol S. K. Leung,

Department of Haematology,

University College London Cancer Institute, University College London,

Paul O'Gorman Building, 72 Huntley

Street, London WC1E 6DD, UK cs.leung@ucl.ac.uk

Specialty section: This article was submitted to Antigen Presenting Cell Biology, a section of the journal Frontiers in Immunology

Received: 30 April 2015

Accepted: 25 August 2015 Published: 09 September 2015

Citation:

Leung CSK (2015) Endogenous antigen presentation of MHC class II epitopes through

non-autophagic pathways.

Front. Immunol. 6:464.

doi: 10.3389/fimmu.2015.00464

\section{Introduction}

Antigenic peptides presented by major histocompatibility complex (MHC) class II molecules were thought to be mainly generated from extracellular sources, and MHC class I peptides were thought to be derived from cytosolic and nuclear proteins. In the classical MHC class II presentation pathway, exogenous antigens are acquired by antigen-presenting cells and delivered into the endo-lysosomal system, where they are broken down to peptides and presented on MHC class II molecules. While this holds true, it has become apparent that non-classical pathways also contribute to MHC class II antigen presentation. Proteins within antigen-presenting cells can endogenously access the MHC class II pathway and their constituent peptide epitopes can be presented to $\mathrm{CD} 4^{+} \mathrm{T}$ cells. The first few studies, shed light on the existence of an endogenous MHC class II pathway, were published in the 1980s (1-3). Jacobson et al. found that target cells endogenously expressed measles virus matrix or nucleocapsid proteins were lysed by class II-restricted measles virus-specific $\mathrm{CD} 4^{+} \mathrm{T}$ cell lines $(4,5)$, indicating cytosolic proteins could be endogenously processed and presented in the MHC class II pathway. Findings from other independent studies also corroborated this by illustrating that some viral proteins were presented by MHC class II molecules via an endogenous processing pathway $(6,7)$. In addition, analysis of purified natural MHC class II ligands has drawn a picture on the origins of antigens presented by MHC class II molecules. A fraction of the peptides bound to MHC class II molecules have been found to be derived from intracellular proteins (8-12). In theory, these intracellular proteins could have been liberated from apoptotic cells and recaptured as exogenous materials by the antigen-presenting cells. Nevertheless, cytosolic epitopes in these studies were isolated from cell lines that were not highly phagocytic, and a high rate of cell death and phagocytosis would be necessary to account for the abundance of some cytosolic epitopes (13). Therefore, despite exogenous antigens being the main source of antigens in the MHC class 
II pathway, endogenous antigens are able to access the MHC class II system. In this review, I will highlight the recent findings on endogenous MHC class II presentation pathways that are not linked to autophagy.

\section{Hijacking the MHC Class I Machinery}

Not long after the discovery of the endogenous MHC class II presentation of antigens, components of the MHC class I pathways, such as proteasome and transporter associated with antigenprocessing complex (TAP), were proposed to contribute to the non-classical presentation of MHC class II epitopes. The proteasome is a multisubunit enzyme complex that degrades a variety of proteins into short polypeptides and amino acids in the cytosol. Its role in endogenous class II presentation has been well documented (14-17). TAP was first shown to be involved in a DR1-restricted presentation and processing of a short cytosolic peptide of the influenza hemagglutinin (18), suggesting that cytosolic peptides could be imported into the endoplasmic reticulum (ER) in a TAP-dependent manner for binding to MHC class II molecules. However, as invariant chain is associated with the MHC class II molecules in the ER to prevent premature loading of peptide, the obstacle has to be overcome in this proposed pathway. Lechler and Aichinger suggested that stress conditions, such as viral infection, could alter the ratio of MHC class II molecules to invariant chain, resulting in the binding of unfolded or partially unfolded proteins to MHC class II molecules. They also proposed that high expression levels of antigen could allow antigen loading in the ER $(19,20)$. Indeed, it has been shown recently that unfolded proteins could compete with invariant chain and bind to MHC class II molecules in the ER before transporting to the cell surface (21). However, the same study did not find evidence that the protein-MHC class II complex on the cell surface could induce $\mathrm{CD} 4^{+} \mathrm{T}$ cell activation. Interestingly, there are numerous examples of CD4 epitopes being presented in the absence of invariant chain expression (22-24), and that invariant chain-negative cancer cells have been used as a means to stimulate tumor-specific T cells (25). Apart from loading the epitopes in the ER, the peptides could be loaded on internalized mature MHC class II molecules through the recycling pathway. In this pathway, the antigens may require proteasome degradation and TAP for delivering the epitopes to the early endosome, where they are loaded onto recycling MHC class II molecules. In fact, it has been demonstrated that endogenous presentation could be mediated through the recycling of MHC class II molecules $(26,27)$. Tewari et al. studied three I-E ${ }^{\mathrm{d}}$ restricted epitopes of PR8 influenza (28) and found two of these epitopes were efficiently generated by a proteasome- and TAPdependent pathway that involved recycling of the MHC class II molecules. Others have shown that MHC class II molecules could internalize and exchange antigenic peptides in the endosomes $(29,30)$, and that the peptide-MHC class II complex could rapidly recycle back to the plasma membrane through a clathrinindependent pathway (31). The conformation of the antigen is crucial for its access to the intracellular processing pathway and binding to the recycling of MHC class II molecules (32), as incompletely folded antigen could lead to selective binding to recycling of MHC class II molecules and result in $\mathrm{CD}^{+}{ }^{+} \mathrm{T}$ cell activation.

\section{Regulation by Heat Shock Protein 90 (HSP90)}

Recent studies on the processing of tumor-associated antigens have uncovered the involvement of components other than the MHC class I machinery in endogenous MHC class II presentation pathways. NY-ESO-1 is a cancer testis antigen expressed in a wide variety of malignant cells and spontaneous immune responses against this antigen have been detected in cancer patients (33). As $\mathrm{CD}^{+}{ }^{+} \mathrm{T}$ cells could directly recognize $\mathrm{MHC}$ class II positive and NY-ESO-1-expressing tumor cells (34), one would then question the underlying mechanism of how this intracellular antigen is processed to $\mathrm{CD} 4^{+} \mathrm{T}$ cells. Tsuji et al. attempted to solve this by studying NY-ESO-1 epitope processing in the melanoma cell line SK-MEL-37 in the presence of various pharmacological inhibitors (35). The processing of the epitope NY-ESO-195-106, restricted through DR01, was inhibited by the proteasome inhibitor epoxomicin but not lactacystin, indicating the processing of this epitope was dependent on the chymotrypsin-like activity of the proteasome. Also, tripeptidyl peptidase II and TAP were involved in degrading the protein and peptide transport. The processing required endosomal protease as treatment with chloroquine and leupeptin inhibited the presentation. However, macroautophagy and chaperone-mediated autophagy were not involved. Instead, the presentation was dependent on endosomal protease and chaperoning by the cytosolic HSP90. This chaperone protein is inducible by stress and is required for the functioning of a large number of client proteins. Both HSP90 inhibitor 17-DMAG and si-RNA-mediated silencing of the protein inhibited the CD4 ${ }^{+}$ $\mathrm{T}$ cell response to epitope NY-ESO-1 ${ }_{95-106}$, indicating the participation of HSP90 in the presentation. HSP90 has also been implicated in direct and cross antigen presentation by professional antigen-presenting cells for $\mathrm{CD}^{+} \mathrm{T}$ cell recognition (36-39); and its role in chaperoning and transferring antigenic peptides to MHC class II molecules in professional antigen-presenting cells has been described $(40,41)$. However, unlike the other NYESO-1 epitope, the DP04-restricted epitope NY-ESO-1 ${ }_{157-170}$ was processed independent of HSP90, as treatment with the HSP90 inhibitors, 17-DMAG and redicicol as well as si-RNA-mediated silencing, had no effect in the presentation (42). This DP04restricted presentation required TAP-mediated peptide transport and endosomal recycling. More studies are required to confirm the role of HSP90 on endogenous processing of MHC class II epitopes.

\section{Internalization from the Plasma Membrane}

Another tumor antigen, gp100, is a melanosomal antigen that has been reported to access the endogenous MHC class II pathway and present efficiently to gp-100-specific $\mathrm{CD} 4^{+} \mathrm{T}$ cells (43). The presentation was inhibited by removing the putative $\mathrm{NH}_{2}$-terminal signal sequence and the last 70 residues in the $\mathrm{COOH}$ terminus in gp100, supporting the important role of melanosomal and endosomal location for efficient MHC class 
II presentation. In the study by Lepage and Lapointe, gp100 transfectants were used to assess the presentation of the DR07restricted epitope gp100 $170-190$. The cell-surface expression of gp100 correlated with MHC class II presentation, suggesting that gp100 might transport to the cell surface before internalizing to relevant endosomal or lysosomal compartments to meet the $\mathrm{MHC}$ class II molecules for presentation. The correlation between gp100 cell-surface expression and the endogenous presentation of protein was also confirmed in another study. Robila et al. analyzed the MHC class II endogenous processing of DR04restricted epitope gp $100_{44-59}$ and demonstrated that endosomal localization and processing by acidic proteases were necessary for gp $100_{44-59}$ presentation (44). The major source of this epitope was from the internalization of the gp100 protein from the plasma membrane through the AP2 adaptor protein. In these studies, although the authors claimed gp100 was endogenously processed in the MHC class II pathway, their experiments could not exclude the possibility that the antigen might have been processed via a conventional exogenous route. As gp100 is a membrane-bound glycoprotein that can be secreted by cells (45), it is possible that MHC class II presentation in these studies did not result from processing of endogenous antigen, but was rather derived from exogenous antigen released from other cells in the culture. This could be easily clarified by a cell-mixing experiment, where gp100 expressing MHC-mismatched target cells are first mixed with MHC-matched target cells lacking the antigen, then co-cultured with gp100-specific $\mathrm{CD}^{+} \mathrm{T}$ cells. If $\mathrm{CD}^{+} \mathrm{T}$ cell response could be detected, then gp100 would be transferred and processed in the conventional manner. In fact, intercellular antigen transfer plays a role in the processing of MHC class II antigens for $\mathrm{CD}^{+}{ }^{+} \mathrm{T}$ cell recognition (46, 47). However, as this pathway involves antigen being secreted and then recaptured as exogenous material by the antigenpresenting cells, this is generally considered to be conventional processing.

\section{References}

1. Bikoff E, Birshtein BK. T cell clones specific for IgG2a of the a allotype: direct evidence for presentation of endogenous antigen. J Immunol (1986) 137(1):28-34

2. Eisenlohr LC, Hackett CJ. Class II major histocompatibility complex-restricted $\mathrm{T}$ cells specific for a virion structural protein that do not recognize exogenous influenza virus. Evidence that presentation of labile $\mathrm{T}$ cell determinants is favored by endogenous antigen synthesis. J Exp Med (1989) 169(3):921-31. doi:10.1084/jem.169.3.921

3. Weiss S, Bogen B. B-lymphoma cells process and present their endogenous immunoglobulin to major histocompatibility complex-restricted T cells. Proc Natl Acad Sci U S A (1989) 86(1):282-6. doi:10.1073/pnas.86.1.282

4. Jacobson S, Sekaly RP, Bellini WJ, Johnson CL, McFarland HF, Long EO. Recognition of intracellular measles virus antigens by HLA class II restricted measles virus-specific cytotoxic T lymphocytes. Ann N Y Acad Sci (1988) 540:352-3. doi:10.1111/j.1749-6632.1988.tb27096.x

5. Jacobson S, Sekaly RP, Jacobson CL, McFarland HF, Long EO. HLA class IIrestricted presentation of cytoplasmic measles virus antigens to cytotoxic $\mathrm{T}$ cells. J Virol (1989) 63(4):1756-62.

6. Jaraquemada D, Marti M, Long EO. An endogenous processing pathway in vaccinia virus-infected cells for presentation of cytoplasmic antigens to class II-restricted T cells. J Exp Med (1990) 172(3):947-54. doi:10.1084/jem. 172.3.947

\section{Conclusion}

This review has summarized the recent studies on endogenous antigen presentation of MHC class II epitopes through non-autophagic pathways. Although different pathways have been described in the non-classical MHC class II processing, there are still questions to be addressed. How are different epitopes selected for different endogenous presentation pathways to $\mathrm{CD} 4^{+} \mathrm{T}$ cells? Why are some epitopes better presented than the others? Despite having the same HLA restriction and epitope in a proximal region within an antigen, antigen-specific $\mathrm{T}$ cells display differential capability to recognize tumor cells. Tsuji et al. has shown that DR01-restricted $\mathrm{CD}^{+}{ }^{+} \mathrm{T}$ cell lines specific for the NY-ESO195-106 epitope were able to respond to un-manipulated melanoma cell lines, while $\mathrm{CD}^{+} \mathrm{T}$ cells specific to NY-ESO-1 ${ }_{87-98}$ could not (35). Another study also reported that two epitopes of the Epstein-Barr virus antigen EBNA1, namely VYG (EBNA1 $\left.{ }_{509-528}\right)$ and PQC (EBNA1 $\left.1_{529-548}\right)$, both restricted through DR11, behaved differently. Whereas VYG-specific $\mathrm{CD} 4{ }^{+} \mathrm{T}$ cells could recognize EBV-positive LCLs directly, it was not the case for the PQC epitope (48). Poor presentation of the latter might be due to the fact that PQC is more prone to lysosomal protease degradation. However, how VYG is endogenously presented in the MHC class II pathway is still not known. In addition, most of these experiments have been in vitro studies; the pathways should be validated in vivo. Gaining a better understanding of these pathways will give insight in the development of vaccines and immunotherapies.

\section{Acknowledgments}

CL was a Croucher Foundation Fellow and is supported by a Research Fellowship from the Swiss National Science Foundation. The author would like to thank Prof. Christian Münz, University of Zürich and Dr. Simon Thomas, University College London, for their critical comments.

7. Nuchtern JG, Biddison WE, Klausner RD. Class II MHC molecules can use the endogenous pathway of antigen presentation. Nature (1990) 343(6253):74-6. doi: $10.1038 / 343074 \mathrm{a} 0$

8. Rudensky A, Preston-Hurlburt P, Hong SC, Barlow A, Janeway CA Jr. Sequence analysis of peptides bound to MHC class II molecules. Nature (1991) 353(6345):622-7. doi:10.1038/353622a0

9. Chicz RM, Urban RG, Gorga JC, Vignali DA, Lane WS, Strominger JL. Specificity and promiscuity among naturally processed peptides bound to HLA-DR alleles. J Exp Med (1993) 178(1):27-47. doi:10.1084/jem.178.1.27

10. Rammensee H, Bachmann J, Emmerich NP, Bachor OA, Stevanovic S. SYFPEITHI: database for MHC ligands and peptide motifs. Immunogenetics (1999) 50(3-4):213-9. doi:10.1007/s002510050595

11. Dongre AR, Kovats S, deRoos P, McCormack AL, Nakagawa T, PaharkovaVatchkova V, et al. In vivo MHC class II presentation of cytosolic proteins revealed by rapid automated tandem mass spectrometry and functional analyses. Eur J Immunol (2001) 31(5):1485-94. doi:10.1002/1521-4141(200105)31: 5<1485::AID-IMMU1485>3.0.CO;2-A

12. Muntasell A, Carrascal M, Serradell L, Veelen Pv PV, Verreck F, Koning F, et al. HLA-DR4 molecules in neuroendocrine epithelial cells associate to a heterogeneous repertoire of cytoplasmic and surface self peptides. J Immunol (2002) 169(9):5052-60. doi:10.4049/jimmunol.169.9.5052

13. Trombetta ES, Mellman I. Cell biology of antigen processing in vitro and in vivo. Annu Rev Immunol (2005) 23:975-1028. doi:10.1146/annurev.immunol.22. 012703.104538 
14. Lich JD, Elliott JF, Blum JS. Cytoplasmic processing is a prerequisite for presentation of an endogenous antigen by major histocompatibility complex class II proteins. J Exp Med (2000) 191(9):1513-24. doi:10.1084/jem.191.9.1513

15. Dani A, Chaudhry A, Mukherjee P, Rajagopal D, Bhatia S, George A, et al. The pathway for MHCII-mediated presentation of endogenous proteins involves peptide transport to the endo-lysosomal compartment. J Cell Sci (2004) 117(Pt 18):4219-30. doi:10.1242/jcs.01288

16. Mukherjee P, Dani A, Bhatia S, Singh N, Rudensky AY, George A, et al. Efficient presentation of both cytosolic and endogenous transmembrane protein antigens on MHC class II is dependent on cytoplasmic proteolysis. J Immunol (2001) 167(5):2632-41. doi:10.4049/jimmunol.167.5.2632

17. Comber JD, Robinson TM, Siciliano NA, Snook AE, Eisenlohr LC. Functional macroautophagy induction by influenza A virus without a contribution to major histocompatibility complex class II-restricted presentation. J Virol (2011) 85(13):6453-63. doi:10.1128/JVI.02122-10

18. Malnati MS, Marti M, LaVaute T, Jaraquemada D, Biddison W, DeMars R, et al. Processing pathways for presentation of cytosolic antigen to MHC class II-restricted T cells. Nature (1992) 357(6380):702-4. doi:10.1038/357702a0

19. Lechler R, Aichinger G, Lightstone L. The endogenous pathway of MHC class II antigen presentation. Immunol Rev (1996) 151:51-79. doi:10.1111/j.1600-065X. 1996.tb00703.x

20. Aichinger G, Lechler RI. Endogenous pathway of class II presentation. Biochem Soc Trans (1995) 23(3):657-60. doi:10.1042/bst0230657

21. Jiang Y, Arase N, Kohyama M, Hirayasu K, Suenaga T, Jin H, et al. Transport of misfolded endoplasmic reticulum proteins to the cell surface by MHC class II molecules. Int Immunol (2013) 25(4):235-46. doi:10.1093/intimm/dxs155

22. Ceman S, Sant AJ. The function of invariant chain in class II-restricted antigen presentation. Semin Immunol (1995) 7(6):373-87. doi:10.1006/smim.1995.0042

23. Viville S, Neefjes J, Lotteau V, Dierich A, Lemeur M, Ploegh H, et al. Mice lacking the MHC class II-associated invariant chain. Cell (1993) 72(4):635-48. doi:10.1016/0092-8674(93)90081-Z

24. Fortin JS, Cloutier M, Thibodeau J. Exposing the specific roles of the invariant chain isoforms in shaping the MHC class II peptidome. Front Immunol (2013) 4:443. doi:10.3389/fimmu.2013.00443

25. Chornoguz O, Gapeev A, O’Neill MC, Ostrand-Rosenberg S. Major histocompatibility complex class II+ invariant chain negative breast cancer cells present unique peptides that activate tumor-specific T cells from breast cancer patients. Mol Cell Proteomics (2012) 11(11):1457-67. doi:10.1074/mcp.M112. 019232

26. Pinet V, Vergelli M, Martin R, Bakke O, Long EO. Antigen presentation mediated by recycling of surface HLA-DR molecules. Nature (1995) 375(6532):603-6. doi:10.1038/375603a0

27. Sinnathamby G, Eisenlohr LC. Presentation by recycling MHC class II molecules of an influenza hemagglutinin-derived epitope that is revealed in the early endosome by acidification. J Immunol (2003) 170(7):3504-13. doi:10. 4049/jimmunol.170.7.3504

28. Tewari MK, Sinnathamby G, Rajagopal D, Eisenlohr LC. A cytosolic pathway for MHC class II-restricted antigen processing that is proteasome and TAP dependent. Nat Immunol (2005) 6(3):287-94. doi:10.1038/ni1171

29. Reid PA, Watts C. Cycling of cell-surface MHC glycoproteins through primaquine-sensitive intracellular compartments. Nature (1990) 346(6285): 655-7. doi: $10.1038 / 346655 \mathrm{a} 0$

30. Pathak SS, Lich JD, Blum JS. Cutting edge: editing of recycling class II:peptide complexes by HLA-DM. J Immunol (2001) 167(2):632-5. doi:10. 4049/jimmunol.167.2.632

31. Walseng E, Bakke O, Roche PA. Major histocompatibility complex class IIpeptide complexes internalize using a clathrin- and dynamin-independent endocytosis pathway. J Biol Chem (2008) 283(21):14717-27. doi:10.1074/jbc. M801070200

32. Mimura Y, Mimura-Kimura Y, Doores K, Golgher D, Davis BG, Dwek RA, et al. Folding of an MHC class II-restricted tumor antigen controls its antigenicity via MHC-guided processing. Proc Natl Acad Sci USA (2007) 104(14):5983-8. doi:10.1073/pnas.0701307104

33. Gnjatic S, Nishikawa H, Jungbluth AA, Gure AO, Ritter G, Jager E, et al. NY-ESO-1: review of an immunogenic tumor antigen. Adv Cancer Res (2006) 95:1-30. doi:10.1016/S0065-230X(06)95001-5
34. Odunsi K, Qian F, Matsuzaki J, Mhawech-Fauceglia P, Andrews C, Hoffman EW, et al. Vaccination with an NY-ESO-1 peptide of HLA class I/II specificities induces integrated humoral and T cell responses in ovarian cancer. Proc Natl Acad Sci U S A (2007) 104(31):12837-42. doi:10.1073/pnas.0703342104

35. Tsuji T, Matsuzaki J, Caballero OL, Jungbluth AA, Ritter G, Odunsi K, et al. Heat shock protein 90-mediated peptide-selective presentation of cytosolic tumor antigen for direct recognition of tumors by CD4(+) T cells. J Immunol (2012) 188(8):3851-8. doi:10.4049/jimmunol.1103269

36. Imai T, Kato Y, Kajiwara C, Mizukami S, Ishige I, Ichiyanagi T, et al. Heat shock protein 90 (HSP90) contributes to cytosolic translocation of extracellular antigen for cross-presentation by dendritic cells. Proc Natl Acad Sci U S A (2011) 108(39):16363-8. doi:10.1073/pnas.1108372108

37. Ichiyanagi T, Imai T, Kajiwara C, Mizukami S, Nakai A, Nakayama T, et al. Essential role of endogenous heat shock protein 90 of dendritic cells in antigen cross-presentation. J Immunol (2010) 185(5):2693-700. doi:10.4049/jimmunol. 1000821

38. Callahan MK, Garg M, Srivastava PK. Heat-shock protein 90 associates with $\mathrm{N}$-terminal extended peptides and is required for direct and indirect antigen presentation. Proc Natl Acad Sci U S A (2008) 105(5):1662-7. doi:10.1073/pnas. 0711365105

39. Kunisawa J, Shastri N. Hsp90alpha chaperones large C-terminally extended proteolytic intermediates in the MHC class I antigen processing pathway. Immunity (2006) 24(5):523-34. doi:10.1016/j.immuni.2006.03.015

40. Houlihan JL, Metzler JJ, Blum JS. HSP90alpha and HSP90beta isoforms selectively modulate MHC class II antigen presentation in B cells. J Immunol (2009) 182(12):7451-8. doi:10.4049/jimmunol.0804296

41. Rajagopal D, Bal V, Mayor S, George A, Rath S. A role for the Hsp90 molecular chaperone family in antigen presentation to $\mathrm{T}$ lymphocytes via major histocompatibility complex class II molecules. Eur J Immunol (2006) 36(4):828-41. doi:10.1002/eji.200535326

42. Matsuzaki J, Tsuji T, Luescher I, Old LJ, Shrikant P, Gnjatic S, et al. Nonclassical antigen-processing pathways are required for MHC class II-restricted direct tumor recognition by NY-ESO-1-specific CD4(+) T cells. Cancer Immunol Res (2014) 2(4):341-50. doi:10.1158/2326-6066.CIR-13-0138

43. Lepage S, Lapointe R. Melanosomal targeting sequences from gp100 are essential for MHC class II-restricted endogenous epitope presentation and mobilization to endosomal compartments. Cancer Res (2006) 66(4):2423-32. doi:10. 1158/0008-5472.CAN-05-2516

44. Robila V, Ostankovitch M, Altrich-Vanlith ML, Theos AC, Drover S, Marks MS, et al. MHC class II presentation of gp100 epitopes in melanoma cells requires the function of conventional endosomes and is influenced by melanosomes. J Immunol (2008) 181(11):7843-52. doi:10.4049/jimmunol.181.11.7843

45. Hoashi T, Tamaki K, Hearing VJ. The secreted form of a melanocyte membranebound glycoprotein (Pmel17/gp100) is released by ectodomain shedding. FASEB J (2010) 24(3):916-30. doi:10.1096/fj.09-140921

46. Taylor GS, Long HM, Haigh TA, Larsen M, Brooks J, Rickinson AB. A role for intercellular antigen transfer in the recognition of EBV-transformed B cell lines by EBV nuclear antigen-specific CD4+ T cells. J Immunol (2006) 177(6):3746-56. doi:10.4049/jimmunol.177.6.3746

47. Reilly M, Mix D, Reilly AA, Ye XY, Winslow GM. Intercellular transfer of a soluble viral superantigen. J Virol (2000) 74(18):8262-7. doi:10.1128/JVI.74.18. 8262-8267.2000

48. Leung CS, Haigh TA, Mackay LK, Rickinson AB, Taylor GS. Nuclear location of an endogenously expressed antigen, EBNA1, restricts access to macroautophagy and the range of CD4 epitope display. Proc Natl Acad Sci U S A (2010) 107(5):2165-70. doi:10.1073/pnas.0909448107

Conflict of Interest Statement: The author declares that the research was conducted in the absence of any commercial or financial relationships that could be construed as a potential conflict of interest.

Copyright (C) 2015 Leung. This is an open-access article distributed under the terms of the Creative Commons Attribution License (CC BY). The use, distribution or reproduction in other forums is permitted, provided the original author(s) or licensor are credited and that the original publication in this journal is cited, in accordance with accepted academic practice. No use, distribution or reproduction is permitted which does not comply with these terms. 